\section{Control of viral latency in neurons by axonal mTOR signaling and the 4E-BP translation repressor}

\author{
Mariko Kobayashi, ${ }^{1}$ Angus C. Wilson, ${ }^{1,2}$ \\ Moses V. Chao, ${ }^{3,4,5,6,7}$ and Ian Mohr ${ }^{1,2,8}$
}

${ }^{1}$ Department of Microbiology, ${ }^{2}$ New York University Cancer Institute, ${ }^{3}$ Molecular Neurobiology Program, Skirball Institute for Biomolecular Medicine, ${ }^{4}$ Department of Cell Biology,

${ }^{5}$ Department of Physiology and Neuroscience, ${ }^{6}$ Department of Psychiatry, ${ }^{7}$ Center for Neural Science, New York University School of Medicine, New York, New York 10016, USA

Latent herpes simplex virus-1 (HSV1) genomes in peripheral nerve ganglia periodically reactivate, initiating a gene expression program required for productive replication. Whether molecular cues detected by axons can be relayed to cell bodies and harnessed to regulate latent genome expression in neuronal nuclei is unknown. Using a neuron culture model, we found that inhibiting mTOR, depleting its regulatory subunit raptor, or inducing hypoxia all trigger reactivation. While persistent mTORC1 activation suppressed reactivation, a mutant 4E-BP (eIF4E-binding protein) translational repressor unresponsive to mTORC1 stimulated reactivation. Finally, inhibiting mTOR in axons induced reactivation. Thus, local changes in axonal mTOR signaling that control translation regulate latent HSV1 genomes in a spatially segregated compartment.

Supplemental material is available for this article.

Received March 1, 2012; revised version accepted June 6, 2012.

The ability to respond to physiological cues in the host neuron and switch between two distinct developmental programs, latency and productive viral replication, is a defining feature of herpes simplex virus (HSV) biology and pathogenesis (Roizman et al. 2007). During latency, the viral genome is maintained as an episome within the neuronal nucleus, and genes required for viral replication are epigenetically silenced (Knipe and Cliffe 2008; Bloom et al. 2010). Thus, viral proteins are not detected, and productive replication is suppressed. In response to incompletely understood physiological cues or changes in immune status, the virus emerges from latency or "reactivates," initiating a complex gene expression program that results in viral replication and culminates in infectious virus production (Knipe and Cliffe 2008). Studies using small animal models have been instrumental

[Keywords: eIF4E-binding protein 4E-BP1; local translational control; mTOR signaling; neurons; virus latency]

${ }^{8}$ Corresponding author

Email ian.mohr@med.nyu.edu

Article is online at http://www.genesdev.org/cgi/doi/10.1101/gad.190157.112. in defining viral gene products involved in reactivation and the role of host innate and acquired immune systems in controlling latency (Wagner and Bloom 1997; Cunningham et al. 2006; Knickelbein et al. 2008; Thompson et al. 2009). While it was first hypothesized in the early 20th century that the virus colonizes peripheral neurons and establishes lifelong latent infections capable of triggering recurrent disease (Cushing 1905; Goodpasture 1929), the molecular signals regulating HSV latency specifically within host neurons, how reactivation is triggered in response to varied environmental stimuli, and how signal transmission occurs from the periphery to repress latent genomes hidden in neuronal nuclei are questions that remain a mystery.

Recently, phosphatidylinositol 3-kinase (PI3-K)/Akt signaling, mediated by nerve growth factor (NGF) binding to the TrkA receptor tyrosine kinase (RTK), was shown to be required to maintain latency and suppress HSV productive (lytic) growth in a primary neuron cell culture model system that faithfully exhibits key hallmarks of latency as defined in animal models (Camarena et al. 2010; Kim et al. 2012; Kobayashi et al. 2012). Even transient interruption of this signaling cascade was sufficient to promote reactivation from latency, suggesting that neuronal PI3-K/Akt signaling targets are likely cellular effectors regulating latency and reactivation. Here, we identify local mTOR signaling in axons as the underlying molecular mechanism whereby a variety of physiological inputs can be integrated to regulate the HSV lytic-latent developmental decision in neurons. We also show that the translational repressor 4E-BP is a key target of $\mathrm{mTORC1}$ signaling that regulates latency. This is the first demonstration that mTOR signaling localized in axons and that subsequent translational regulation via 4E-BP controls expression of latent viral genomes in neuronal nuclei. Furthermore, it provides a new framework to understand how physical and metabolic stresses can interrupt HSV latency at the neuron-autonomous level through mTOR.

\section{Results and Discussion}

The cellular mTORC1 kinase is a key target of the PI3-K/ Akt pathway that plays important roles in neuronal functions, including control of synaptic plasticity, longterm potentiation (LTP), axonal growth, and regeneration (Willis and Twiss 2006; Lin and Holt 2008; Richter and Klann 2009|. These processes require protein synthesis, which can be potentially regulated by the mTORC1 substrates p70 S6K and eIF4E-binding proteins (4E-BPs) (Ma and Blenis 2009).

To determine whether mTOR signaling regulates reactivation, cultured rat sympathetic neurons latently infected with an HSV1-EGFP reporter were pulse-treated with the active site mTOR inhibitor PP242, with rapamycin, or with the DMSO vehicle. Infectious virus production and EGFP expression are not detected in latently infected cultures prior to application of the reactivation stimulus (Camarena et al. 2010; Kobayashi et al. 2012). While rapamycin is selective for mTORC1, PP242 inhibits both of the mTOR-containing complexes mTORC1 and mTORC2, the latter of which activates Akt (Feldman et al. 2009). Under these conditions, treating latently 
infected cultures with PP242 or rapamycin prevented inactivation of the 4E-BP translational repressor by mTORC1-mediated phosphorylation (Fig. 1A,B) and stimulated accumulation of the viral ICP27 mRNA, which encodes an essential lytic activator, to well above levels detected in DMSO control-treated cultures (Fig. $1 \mathrm{C})$. To assess reactivation via EGFP reporter gene expression or infectious virus production, rapamycin or PP242 was applied for $20 \mathrm{~h}$ and then removed. Stable
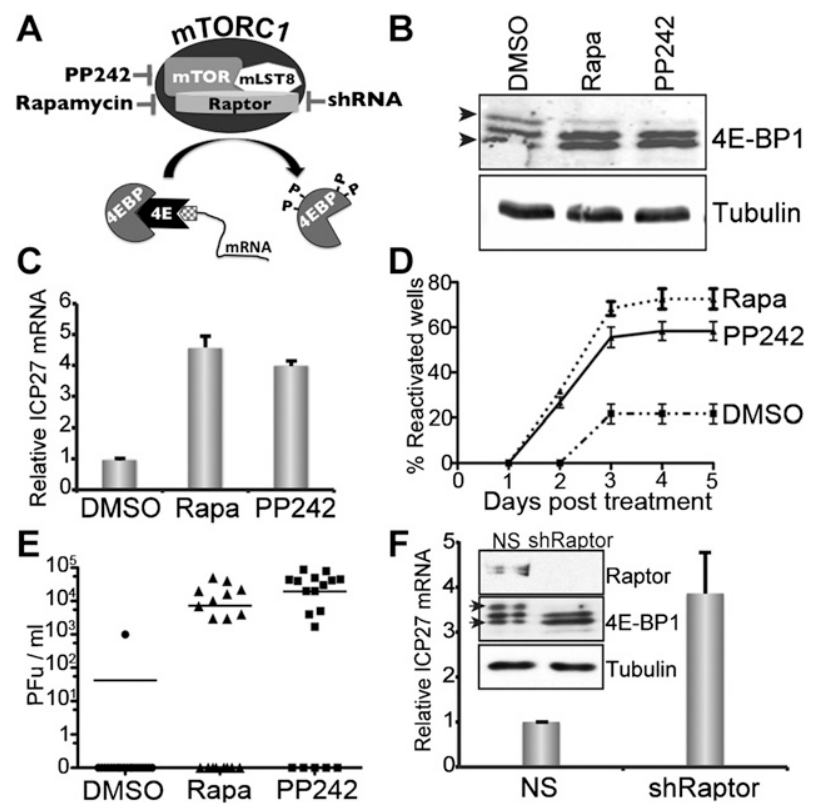

Figure 1. Interfering with mTOR signaling in neurons induces reactivation of latent HSV1. (A) Activation of mTORC1 by neurotrophic factor and RTK-mediated PI3-K/Akt signaling controls the translational repressor 4E-BP. Rapamycin, PP242, and raptor shRNA independently inhibit $\mathrm{mTORC1}$, preventing inactivation of the translation repressor 4E-BP1 by hyperphosphorylation and suppressing cap-dependent mRNA translation. $(B)$ After treatment with rapamycin (rapa; $100 \mathrm{nM}$ ) or PP242 (2.5 $\mathrm{MM}$ ) for $20 \mathrm{~h}$, total protein isolated from latently infected neurons was fractionated by SDSPAGE in a $17.5 \%$ gel and analyzed by immunoblotting. Slowmigrating hyperphosphorylated (top arrow) and faster-migrating hypophosphorylated (bottom arrow) 4E-BP1 forms are indicated at the left. Tubulin immunoblot provides a loading control. $(C) \mathrm{mTOR}$ pharmacological inhibitors induce HSV1 reactivation. Latently infected neurons were treated with PP242 or rapa for $20 \mathrm{~h}$, and viral ICP27 mRNA levels were quantified by quantitative RT-PCR (qRTPCR). (D) As in $C$, except after $20 \mathrm{~h}$, medium was removed and immediately replaced with fresh medium lacking inhibitor. EGFPpositive wells resulting from expression of the late virus lytic Us11EGFP fusion protein were quantified over $5 \mathrm{~d}$. Typically, 10\%-20\% of infected cultures spontaneously reactivate as assessed by EGFP expression. Each data point represents 20 individual wells of a 96well plate. Error bars indicate standard error of the mean (SEM). (E) Infectious virus production was evaluated by plaque assay after $5 \mathrm{~d}$. The bar shows the average number of plaque-forming units (PFU) from 20 wells. $(F)$ Cultures latently infected with EGFP-expressing HSV1 and transduced with a lentivirus expressing an shRNA targeting the mTORC1-specific subunit raptor or a NS control were established in the presence of acyclovir (ACV) as described (Camarena et al. 2010; Kobayashi et al. 2012). After 6 d, ACV was removed, and $20 \mathrm{~h}$ later, total RNA was isolated. The abundance of mRNA encoding the lytic HSV regulator ICP27 was quantified by qRT-PCR and normalized to levels detected in NS control shRNA transduced cultures. Error bars indicate SEM. (Inset) Total protein from neurons transduced with the indicated shRNA-expressing lentivirus was analyzed by immunoblotting after $5 \mathrm{~d}$. treatment with either compound suppressed protein synthesis required for EGFP detection and productive viral replication (Supplemental Fig. S1). While only background spontaneous reactivation levels, evidenced by EGFP detection (Fig. 1D) and infectious virus production (Fig. 1E), were observed in DMSO control-treated cultures, transient PP242 or rapamycin exposure efficiently induced reactivation.

The control of latency by mTORC1 signaling was further investigated using RNAi to deplete raptor, an mTORC1-specific regulatory subunit responsible for complex assembly and substrate selectivity (Sengupta et al. 2010). Lentiviruses expressing control, nonsilencing (NS) shRNA, or a raptor-specific shRNA (Thoreen et al. 2009) were delivered into latently infected neurons. The raptor shRNA effectively depleted endogenous raptor, suppressed phosphorylation of the mTORC1 substrate 4E-BP1 compared with the NS control, and induced expression of the critical HSV1 lytic regulator ICP27, indicative of reactivation (Fig. 1F). Together, experimental results using three independent means to antagonize mTORC1 demonstrate that continuous mTORC1 activity is required to suppress lytic replication and maintain HSV1 latency in neurons.

mTORC1 activation is also controlled by the small GTPase rheb (ras homolog enriched in brain) (Sengupta et al. 2010). To determine whether persistent mTORC1 activation is both necessary and sufficient to maintain latency, epitope-tagged mutant rheb protein (Q64L) was expressed in latently infected neurons using a doxycycline (dox)-inducible lentivirus. The Q64L rheb has reduced GTPase activity (Li et al. 2004; Long et al. 2005) and behaves as a constitutive mTORC1 activator upon doxinduced expression (Fig. 2A,B). Reactivation was assessed after treatment with the PI3-K inhibitor LY294002 (Fig. 2C); the Akt inhibitor VIII (AKT VIII) (Fig. 2D), a potent allosteric inhibitor that suppresses regulatory phosphorylation marks on Akt (Calleja et al. 2009); or the mTORC1-selective inhibitor rapamycin \pm dox. Akt S473 phosphorylation was not detected in LY294002- or AKT VIII-treated cultures, verifying the efficacy of these inhibitors. In addition, induction of rhebQ64L stimulated 4E-BP1 hyperphosphorylation (Fig 2B, cf. lanes 2 and 3, lanes 5 and 6), confirming rheb-mediated mTORC1 activation without detectable Akt activation. Significantly, rhebQ64L induction suppressed reactivation induced by LY294002 (Fig. 2C) or AKT VIII (Fig. 2D), establishing that persistent $\mathrm{mTORC} 1$ activation is required to maintain latency. In contrast, 4E-BP1 remained predominately hypophosphorylated in rapamycin-treated cultures following dox induction of HA-rhebQ64L (Fig 2B, cf. lanes 8 and 91, consistent with rapamycin acting downstream from rheb. Importantly, rhebQ64L induction did not detectably prevent rapamycin-induced reactivation (Fig. 2E). This established that continuous mTORC1 signaling, a critical NGF-responsive regulator of neuronal homeostasis, was necessary and sufficient to maintain HSV1 latency in cultured neurons.

NGF removal stimulates HSV1 reactivation in an animal model, explanted ganglia, and cultured neurons (Wilcox and Johnson 1987; Hill et al. 1997; Du et al. 2011). Many consequences of neurotrophin signaling on target neurons require protein synthesis, including cell death induced by NGF withdrawal (Martin et al. 1988, 1992; Franklin and Johnson 1998). Continuous protein synthesis 
A

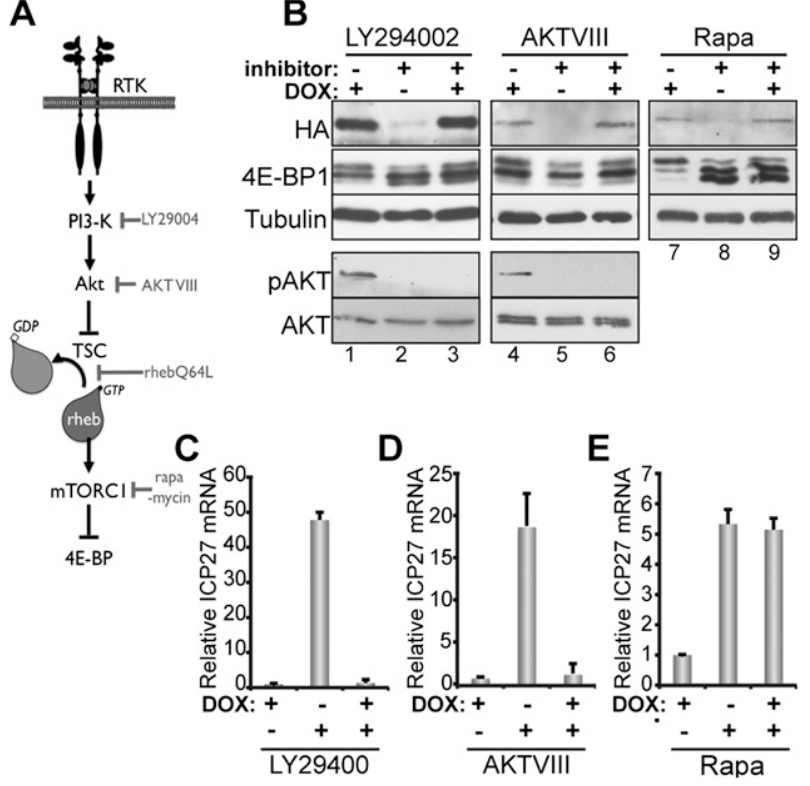

Figure 2. Persistent rheb-mediated mTORCl activation is sufficient to maintain latency and prevent inducible reactivation. $(A \mid$ Diagram of mTORC1 signaling induced by NGF-mediated TrkA/ PI3-K/AKT pathway activation. The receptor RTK for NGF is TrkA. (TSC) Tuberous sclerosis complex; (Rheb) rheb depicted in GDP- and GTP-bound forms. Chemical inhibitors or mutant proteins (rhebQ64L) act at the point shown at the right of the diagram (in gray). $(B-E)$ Latently infected neurons were transduced with a lentivirus encoding HA-tagged rheb (Q64L) expressed from a dox-inducible promoter. Twenty-four hours later, ACV was removed and replaced with medium containing $3 \mu \mathrm{M}$ dox. After $48 \mathrm{~h}$, cultures were treated with an inhibitor (10 $\mu$ M LY294002, $5 \mu$ M AKT VIII, or 100 $\mathrm{nM}$ rapamycin). (B) Total protein was isolated $24 \mathrm{~h}$ after inhibitor addition, fractionated by SDS-PAGE, and analyzed by immunoblotting. Total AKT and tubulin immunoblots are loading controls. pAKT is a phospho-specific Akt S473 antibody. Note that each drug treatment panel represents an independent experiment on separate immunoblots. Thus, comparing relative levels of HA-Rheb expression between LY294002-, AKT VIII-, and rapamycin-treated cultures is not possible. $(C-E)$ Total RNA was collected $20 \mathrm{~h}$ after inhibitor addition, and the abundance of mRNA encoding the HSV essential lytic activator ICP27 was measured by qRT-PCR. Error bars indicate SEM.

is also required to maintain latency, as transient $(3-\mathrm{h})$ inhibition of protein synthesis induced reactivation (Supplemental Fig. S2; Wilcox et al. 1990). Notably, PI3-K signaling via $\mathrm{mTORC} 1$ is a physiological regulator of cell protein synthesis (Fig. 2A; Ma and Blenis 2009; Sengupta et al. 2010). To determine whether environmental signals that regulate protein synthesis via mTORC1 influence the HSV1 lytic-latent decision, latently infected cultures were maintained under normoxia $\left(20 \% \mathrm{O}_{2}\right)$ or hypoxia $\left(1 \% \mathrm{O}_{2}\right)$. Unlike puromycin or cycloheximide, which inhibit nearly all protein synthesis (Supplemental Fig. S2), hypoxia selectively inhibits cap-dependent mRNA translation by preventing mTORCl-mediated hyperphosphorylation of the 4E-BP translational repressor (Brugarolas et al. 2004; Connolly et al. 2006). Importantly, hypoxia was nearly as effective in stimulating reactivation as a puromycin pulse in normoxic cultures (Fig. 3A-C). This implies that hypoxia induces reactivation by inhibiting mTORC1-stimulated cap-dependent mRNA translation.
Two mTORC1 substrates, p70 S6 kinase (p70 S6K) and 4E-BP1, have separate roles controlling protein synthesis. Whereas p70 S6K is activated upon phosphorylation by mTORC1, 4E-BP1 is inhibited. Besides phosphorylating ribosomal protein $\mathrm{S} 6$, p70 $\mathrm{S} 6 \mathrm{~K}$ stimulates translation elongation by inactivating eEF2 kinase and promotes initiation by inactivating the eIF4A inhibitor PDCD4. Alternatively, 4E-BP1 selectively represses cap-dependent translation by associating with the cap-binding initiation factor eIF4E and inhibiting 40S ribosome recruitment to mRNA (Ma and Blenis 2009). 4E-BP phosphorylation on T37/46 by mTORC1 inactivates 4E-BP (Gingras et al. 1999). To prevent 4E-BP inactivation, an epitope-tagged T37A/T46A 4E-BP1 double mutant (AA) was introduced into latently infected neurons using a dox-inducible lentivirus and reactivation \pm dox compared with cultures expressing a wild-type 4E-BP1 control (Fig. 4A). While wild-type 4E-BP induction only modestly effected protein synthesis and did not detectably induce reactivation, AA 4E-BP expression suppressed translation by $\sim 40 \%$ and was as effective as rapamycin in inducing reactivation (Fig. 4B,C). Not only does this establish that a constitutively active 4E-BP translation repressor induces reactivation, it shows for the first time that conditional expression of a single cellular gene can alter the viral latent state and trigger reactivation. This effect was selective for the mTORC1 substrate 4E-BP1, as S6K1 depletion

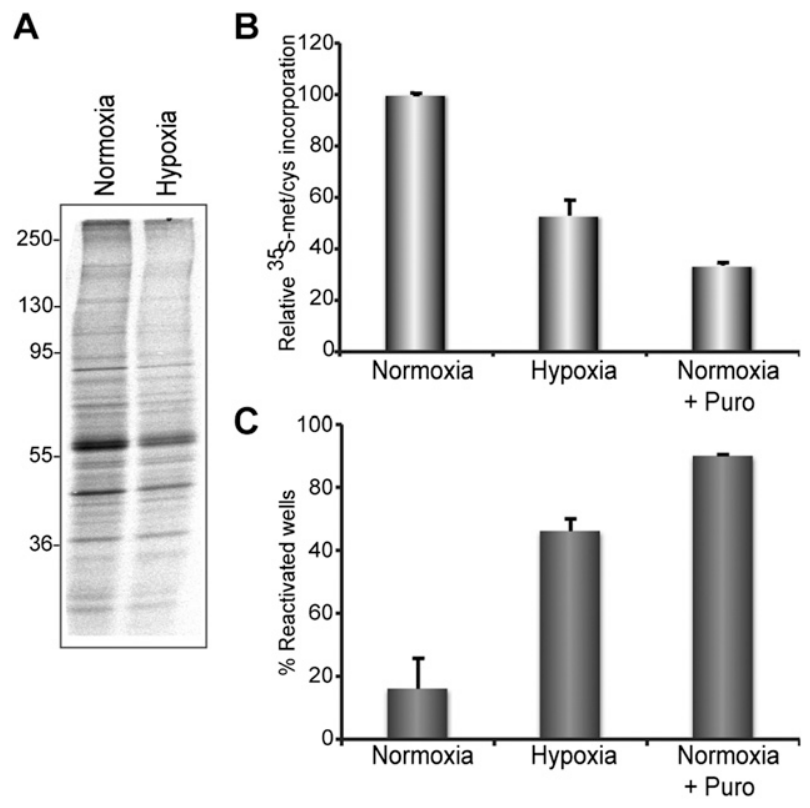

Figure 3. Hypoxic stress and inhibition of cap-dependent mRNA translation trigger inducible reactivation. $(A, B)$ Suppression of protein synthesis by hypoxia in latently infected neurons. After $69 \mathrm{~h}$ in $1 \% \mathrm{O}_{2}$, cultures were metabolically labeled with ${ }^{35} \mathrm{~S}$ amino acids for $3 \mathrm{~h}$ in $1 \% \mathrm{O}_{2}$. Control cultures continuously maintained under normoxia $\left(\sim 20 \% \mathrm{O}_{2}\right)$ were labeled in parallel. Total protein from hypoxic $\left(1 \% \mathrm{O}_{2}\right)$ and normoxic cultures was isolated, fractionated by SDS-PAGE, and analyzed by autoradiography $(A)$, and the relative amounts of acid-insoluble radioactivity were quantified by liquid scintillation counting $(B)$. Puromycin (puro) treatment of normoxic cultures for $3 \mathrm{~h}$ provided a positive control for inhibiting protein synthesis. (C) After $72 \mathrm{~h}$ in hypoxia, the percentage of EGFP-positive, reactivated wells was compared with normoxic cultures. Puro treatment of normoxic cultures for $3 \mathrm{~h}$ provided a positive control for inducible reactivation. 


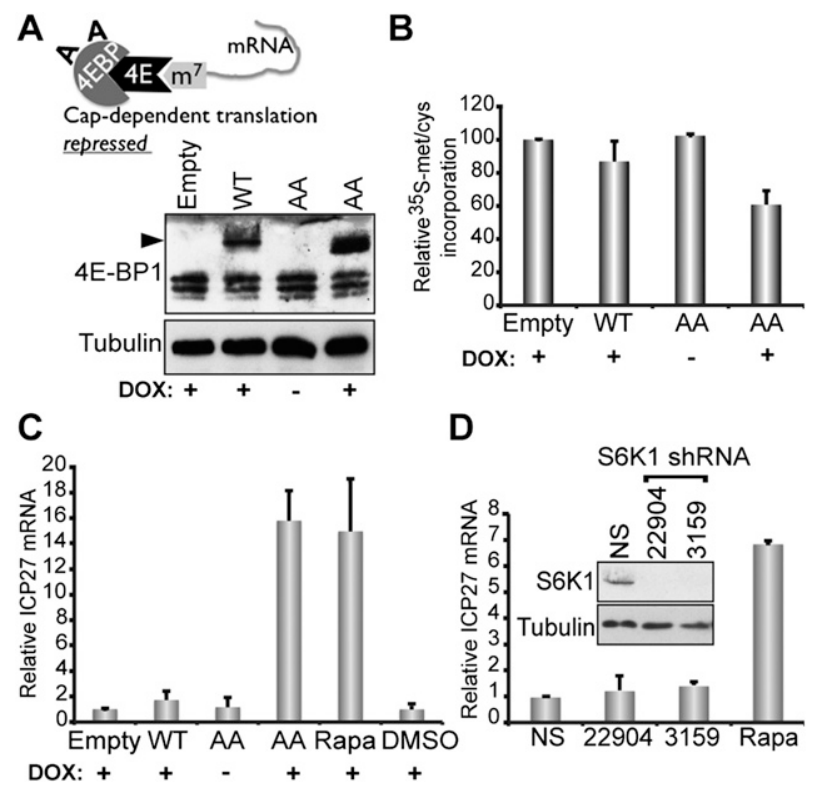

Figure 4. Inducible expression of a constitutively active 4E-BP1 repressor promotes reactivation. (A) The 4E-BP1 (AA) mutant is unresponsive to $\mathrm{mTORC} 1$-mediated negative regulation and constitutively sequesters eIF4E (4E), suppressing cap-dependent mRNA translation. $\left(\mathrm{m}^{7}\right) \mathrm{m}^{7} \mathrm{GTP}$ cap at the mRNA $5^{\prime}$ end. Latently infected neurons were transduced with a dox-inducible lentivirus encoding HA-tagged 4E-BP1 wild-type (WT), AA, or empty vector. After dox induction, total protein was analyzed by immunoblotting as described in Figure 2B. The black arrowhead at the left of the panel denotes slower-migrating (HA)-tagged 4E-BP1; endogenous 4E-BP1 migrates faster. $(B)$ Total protein was isolated from cultures metabolically labeled with ${ }^{35} \mathrm{~S}$ amino acids for $3 \mathrm{~h}$, and the relative levels of radioactivity incorporated compared with the empty vector control were determined. (C) RNA was collected $72 \mathrm{~h}$ after dox induction; ICP2 7 mRNA levels were quantified by qRT-PCR and normalized to cultures transduced with empty vector control. Rapamycin (100 nM) and DMSO-treated cultures were not transduced with lentivirus and, respectively, serve as \pm reactivation controls. Error bars indicate SEM. (D) Cultures latently infected with EGFP-expressing HSV1 and transduced with one of two lentiviruses (22904 and 3159) expressing shRNA that depleted p70 S6K1 or a NS shRNA control were established in the presence of ACV as described (Camarena et al. 2010; Kobayashi et al. 2012). After $6 \mathrm{~d}$, ACV was removed, and $20 \mathrm{~h}$ later, total RNA was isolated. ICP27 mRNA levels were quantified by qRT-PCR as in C. (Inset) Immunoblot of neuronal lysates validating S6K1 silencing. Tubulin is a loading control. Rapamycin was included as a positive control.

did not detectably induce reactivation (Fig. 4D). Even though S6K1 substrates can control translation, many, like eEF2 kinase, eIF4B, and rps6, are also phosphorylated by S6K2 and p90 RSK, allowing S6K1 depletion to be tolerated (Ma and Blenis 2009; Magnuson et al. 2012). Accordingly, S6K1 depletion did not detectably suppress global protein synthesis in neurons (Supplemental Fig. S3).

To relay signals from RTK mediators like mTORC1 over long distances that separate axon terminals from neuronal cell bodies, neurotrophins such as NGF use axonal transport mechanisms (Chao 2003). Translation of pre-existing mRNAs within discrete subcellular compartments is stimulated in response to local neurotrophin signaling. Local translation allows for rapid responses and plays a critical role regulating neuronal homeostasis (Willis and Twiss 2006; Lin and Holt 2008; Richter and Klann 2009|. Moreover, subsequent signaling or trafficking of proteins produced locally in axons can influence processes elsewhere in the neuron, including the nucleus (Cox et al. 2008). Having established that latent HSV1 can respond to a generalized inhibition of mTORC1 (Fig. 1), we asked whether latent virus can sense a localized rather than global interruption of neuronal signaling. To investigate whether spatial-specific parameters could control HSV latency in neurons, a compartmentalized culture system was used to physically separate axons from their cell bodies (Zheng et al. 2001; Cox et al. 2008). By isolating axons from cell bodies in discrete chambers, each compartment can be independently manipulated. Neurons were plated onto a semiporous membrane in a Boyden chamber cell culture insert and submerged in wells containing medim + NGF (Jeanneteau et al. 2010). The resulting NGF gradient allowed penetration of axons through the pores and their growth along the membrane underside, establishing the lower, "axon-only" compartment (Fig. 5A). After establishing latent HSV1 infections in compartmentalized cultures, PP242 was applied to the cell body and/or axonal compartment for $30 \mathrm{~min}$. Medium was subsequently removed from both compartments and immediately replaced with fresh medium lacking inhibitor. When added only to the axon compartment, PP242 effectively inhibited 4E-BP1 hyperphosphorylation in isolated axons but did not detectably inhibit mTORC1mediated 4E-BP1 phosphorylation in the cell body chamber (Fig. 5B). In contrast, PP242 addition to both top and bottom chambers effectively inhibited 4E-BP1 phosphorylation in neurons plated in the top compartment (Fig. 5B). Remarkably, axonal PP242 application was as effective at stimulating reactivation as global inhibition of mTORC1 signaling (Fig. 5C,D). While Boyden chambers permit harvesting axons for biochemical analysis, isolation between the somal and axonal compartments may not be absolute. To achieve a more stringent fluidic isolation of axons from the soma, the experiment was repeated using a microfluidic chamber where diffusion from one compartment to another is blocked by hydrostatic pressure (Taylor et al. 2005). Once again, a PP242 pulse in the axon compartment potently induced reactivation of latent genomes sequestered in the isolated soma compartment (Fig. 5D-F). Altogether, this demonstrates that latent HSV genomes resident in the neuronal soma can respond to localized changes in mTOR signaling in axons.

Localized changes of neuronal mTORC1 activity can regulate mRNA translation, growth cone formation following axonal injury, axonal guidance, LTP, and calcium signaling (Willis and Twiss 2006; Lin and Holt 2008; Richter and Klann 2009). Here, we establish that mTORC1 regulates the lytic/latent switch of the neurotrophic herpes virus HSV1 and show that latent genomes within nuclei reactivate in response to axonal cues, providing direct experimental evidence that axons can monitor environmental signals to control expression profiles of repressed genomes in cell bodies. Axonal mTOR signaling thus provides an attractive molecular mechanism to explain how nerve endings innervating epithelium detect stimuli that directly control latency and reactivation at a distant site; namely, within the neuronal nucleus located in the trigeminal ganglion.

As a central node sampling fundamental homeostasis indicators, including the availability of growth factors, amino acids, glucose, energy, and oxygen and the pres- 
A
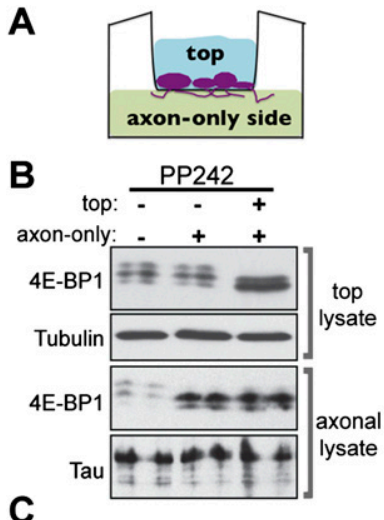

C

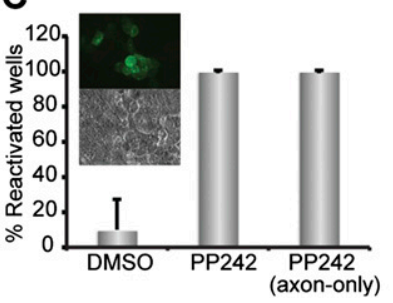

D

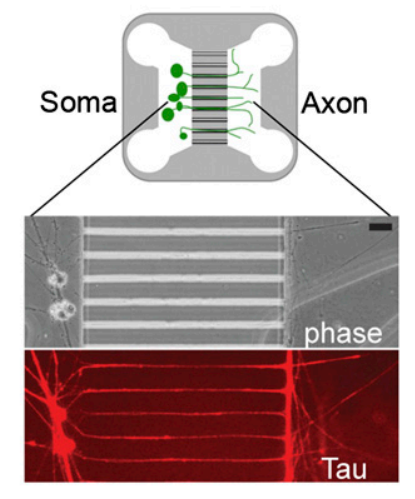

E

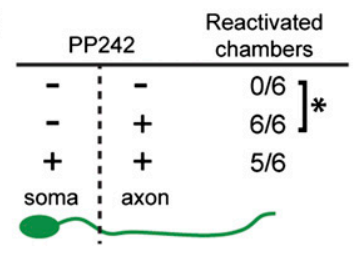

Figure 5. Inhibiting axonal $\mathrm{mTOR}$ signaling triggers reactivation of latent genomes sequestered in neuronal cell bodies. $(A)$ Creating an "axon-only" compartment using Boyden chamber inserts. Neurons are plated onto a membrane with $1-\mu \mathrm{m}$ pores in the top compartment. High NGF in the bottom compartment causes axons to grow through the pores into the lower chamber, establishing an axon-only side. Cell bodies are too large to pass through the pores. $(B)$ Lysates from the top and axon-only compartments ( \pm PP242) were analyzed by immunoblotting. $(C)$ Reactivation assay depicting the percentage of EGFP-positive wells after DMSO or $2.5 \mu \mathrm{M}$ PP242 treatment of top versus axon-only chambers. The graph represents collective analysis of three independent experiments with each point derived from eight Boyden chambers. Error bars indicate SEM. (Inset) Reactivated neurons detected by fluorescent imaging of cell bodies in the top chamber. $(D)$ Schematic of a microfluidic device for separating somal and axonal compartments. Neurons are drawn in green. Phase and immunofluorescence (Tau) images show axons crossing the microgrooves separating the two compartments. Bar, 40 $\mu \mathrm{m}$. (E) The fraction of EGFP-positive, reactivated microfluidic chambers in response to $2.5 \mu \mathrm{M}$ PP242 treatment ( \pm ) of somal versus axonal compartments for $1 \mathrm{~h}$ are shown. $\left(^{\star}\right) P \leq 0.0039$.

ence of DNA damage, mTORC1 is ideally positioned to govern the viral life cycle. In response to this diverse array of inputs, mTORCl coordinates and promotes discrete physiological outputs that regulate autophagy, cell growth, translation, metabolism, and mitochondrial function (Sengupta et al. 2010). Much in the same way, adoption of lytic or lysogenic growth pathways by bacteriophage $\lambda$ relies on essential host functions to provide a rheostat for nutrient availability (Herman et al. 1993).

While productive replication of many viruses activates or inhibits $\mathrm{mTORC} 1$ to stimulate or repress cap-dependent translation in acutely infected cells (Walsh and Mohr 2011), this represents the first example of a virus conscripting mTOR as a sensor to monitor environmental conditions and regulate the latent-lytic developmental decision. Proteins whose synthesis is regulated by mTORC1 and the 4E-BP translational repressor likely contribute to controlling latency at the level of the viral episome in a neuron cell-autonomous manner. Conceivably, these proteins could indirectly or directly regulate epigenetic chromatin marks, affect levels of virus-encoded microRNAs that antagonize lytic gene expression (Umbach et al. 2008), or control subcellular localization of HCF-1, a key cellular factor required for transcriptional activation of viral genes (Kim et al. 2012). Identification of these proteins and their mechanisms of action could lead to new therapeutic strategies targeting the latent genome reservoir.

\section{Materials and methods}

\section{Cell culture and latent infection of primary neurons}

Latent infections were established in superior cervical ganglia (SCG) neurons using wild-type HSV1 (Patton strain) expressing an EGFP-Us11 fusion protein as described (Camarena et al. 2010; Kobayashi et al. 2012). Standard reactivation assays were performed in triplicate using 96-well plates with a sample size of 20 wells. Latent infections in the Boyden chambers (Millipore 24-well Millicell inserts, $1.0-\mu \mathrm{m}$ pore) were established by plating $\sim 10,000$ cells in the top compartment in the presence of NGF (50 $\mu \mathrm{g} / \mathrm{mL})$, reducing NGF to $20 \mu \mathrm{g} / \mathrm{mL}$ at day 3 in vitro (DIV3), and removing it entirely by DIV4, while maintaining normal culture conditions on the axon-only side at all times. Cultures were pretreated with acyclovir (ACV) at DIV4, latently infected with HSV1 from the top compartment at DIV5, and induced to reactivate on DIV10. Each experiment was performed at least three times. Latent infections using microfluidic devices (Xona Microfluidics no. SND450) were established by plating $\sim 40,000$ SCG neurons in the cell body (somal) chamber on glass coverslips coated with $2 \mu \mathrm{g} / \mathrm{mL}$ laminin and $200 \mu \mathrm{g} / \mathrm{mL}$ poly D-lysine (Sigma no. L2020 and P0899) in the presence of $100 \mathrm{ng} / \mathrm{mL}$ NGF in both somal and axonal compartments. NGF concentration in the somal compartment was reduced from $100 \mathrm{ng} / \mathrm{mL}$ to $5 \mathrm{ng} / \mathrm{mL}$ on DIV3 then raised to $100 \mathrm{ng} / \mathrm{mL}$ on DIV6. This facilitates axon growth from the somal to the axonal compartment, where the NGF concentration is higher. On DIV7, somal compartments were infected with HSV1 (multiplicity of infection $[\mathrm{MOI}]=2$ ) in the presence of $100 \mu \mathrm{M}$ ACV and induced to reactivate on DIV14.

\section{Real-Time quantitative RT-PCR}

RNA isolated using Trizol from 10,000 SCG neurons per sample was quantified using a NanoDrop spectrophotometer. RT was performed to obtain first strand cDNA using qScript cDNA Supermix (Quanta Biosciences). Real-time PCR was performed using the Bio-Rad iCycler iQ system with IQ SYBR Green Supermix (Bio-Rad). Each fluorescence cycle threshold $\left(\mathrm{C}_{t}\right)$ value obtained using HSV1 ICP27 primers was normalized against that of $18 \mathrm{~S}$ rRNA. Three independently isolated RNA samples were analyzed for each condition.

\section{Acknowledgments}

We thank Katrin Deinhardt for invaluable advice concerning compartmentalized neuronal cultures, Sean Hagerty for the rheb construct, and Kevan Shokat for PP242. This work was supported by grants to M.V.C. (NS21072 and HD23315), A.C.W. (GM61139 and S10RR017970), and I.M. (AI073898 and GM056927) from the NIH. M.K. acknowledges support from T32AI007647 and T32AI07180.

\section{References}

Bloom DC, Giordani NV, Kwiatkowski DL. 2010. Epigenetic regulation of latent HSV-1 gene expression. Biochim Biophys Acta 1799: 246-256. Brugarolas J, Lei K, Hurley RL, Manning BD, Reiling JH, Hafen E, Witters LA, Ellisen LW, Kaelin WG Jr. 2004. Regulation of mTOR function in response to hypoxia by REDD1 and the TSC1/TSC2 tumor suppressor complex. Genes Dev 18: 2893-2904.

Calleja V, Laguerre M, Parker PJ, Larijani B. 2009. Role of a novel PHkinase domain interface in $\mathrm{PKB} /$ Akt regulation: Structural mechanism for allosteric inhibition. PLoS Biol 7: e17. doi: 10.1371/journal. pbio. 1000017.

Camarena V, Kobayashi M, Kim JY, Roehm P, Perez R, Gardner J, Wilson AC, Mohr I, Chao MV. 2010. Nature and duration of growth factor 
signaling through receptor tyrosine kinases regulates HSV-1 latency in neurons. Cell Host Microbe 8: 320-330.

Chao MV. 2003. Retrograde transport redux. Neuron 39: 1-2.

Connolly E, Braunstein S, Formenti S, Schneider RJ. 2006. Hypoxia inhibits protein synthesis through a 4E-BP1 and elongation factor 2 kinase pathway controlled by mTOR and uncoupled in breast cancer cells. Mol Cell Biol 26: 3955-3965.

Cox LJ, Hengst U, Gurskaya NG, Lukyanov KA, Jaffrey SR. 2008. Intraaxonal translation and retrograde trafficking of CREB promotes neuronal survival. Nat Cell Biol 10: 149-159.

Cunningham AL, Diefenbach RJ, Miranda-Saksena M, Bosnjak L, Kim M, Jones C, Douglas MW. 2006. The cycle of human herpes simplex virus infection: Virus transport and immune control. J Infect Dis 194: S11-S18. doi: $10.1086 / 505359$.

Cushing H. 1905. The surgical aspects of major neuralgia of the trigeminal nerve. JAMA 44: 773-779.

Du T, Zhou G, Roizman B. 2011. HSV-1 gene expression from reactivated ganglia is disordered and concurrent with suppression of latencyassociated transcript and miRNAs. Proc Natl Acad Sci 108: 1882018824.

Feldman ME, Apsel B, Uotila A, Loewith R, Knight ZA, Ruggero D, Shokat KM. 2009. Active-site inhibitors of mTOR target rapamycinresistant outputs of mTORC1 and mTORC2. PLOS Biol 7: e38. doi: 10.1371/journal.pbio.1000038.

Franklin JL, Johnson EM. 1998. Control of neuronal size homeostasis by trophic factor-mediated coupling of protein degradation to protein synthesis. J Cell Biol 142: 1313-1324.

Gingras AC, Gygi SP, Raught B, Polakiewicz RD, Abraham RT, Hoekstra MF, Aebersold R, Sonenberg N. 1999. Regulation of 4E-BP1 phosphorylation: A novel two-step mechanism. Genes Dev 13: 1422-1437.

Goodpasture EW. 1929. Herpetic infection with especial reference to involvement of the nervous system. Medicine 8: 223-243.

Herman C, Ogura T, Tomoyasu T, Hiraga S, Akiyama Y, Ito K, Thomas R, D'Ari R, Bouloc P. 1993. Cell growth and 1 phage development controlled by the same Escherichia coli gene, ftsH/hflB. Proc Natl Acad Sci 90: 10861-10865.

Hill JM, Garza HH Jr, Helmy MF, Cook SD, Osborne PA, Johnson EM Jr, Thompson HW, Green LC, O'Callaghan RJ, Gebhardt BM. 1997. Nerve growth factor antibody stimulates reactivation of ocular herpes simplex virus type 1 in latently infected rabbits. J Neurovirol 3: 206211.

Jeanneteau F, Deinhardt K, Miyoshi G, Bennett AM, Chao MV. 2010. The MAP kinase phosphatase MKP-1 regulates BDNF-induced axon branching. Nat Neurosci 13: 1373-1379.

Kim JY, Mandarino A, Chao M, Mohr I, Wilson AC. 2012. Transient reversal of episome silencing precedes VP16-dependent transcription during reactivation of latent HSV-1 in neurons. PLoS Pathog 8: e1002540. doi: 10.1371/journal.ppat.1002540.

Knickelbein JE, Khanna KM, Yee MB, Baty CJ, Kinchington PR, Hendricks RL. 2008. Noncytotoxic lytic granule-mediated CD8 ${ }^{+}$ T cell inhibition of HSV-1 reactivation from neuronal latency. Science 322: $268-271$.

Knipe DM, Cliffe A. 2008. Chromatin control of herpes simplex virus lytic and latent infection. Nat Rev Microbiol 6: 211-221.

Kobayashi M, Kim JY, Camarena V, Roehm PC, Chao MV, Wilson AC, Mohr I. 2012. A primary neuron culture system for the study of herpes simplex virus latency and reactivation. J Vis Exp 62: e3823. doi: $10.3791 / 3823$.

Li Y, Inoki K, Guan KL. 2004. Biochemical and functional characterizations of small GTPase Rheb and TSC2 GAP activity. Mol Cell Biol 24: 7965-7975.

Lin AC, Holt CE. 2008. Function and regulation of local axonal translation. Curr Opin Neurobiol 18: 60-68.

Long X, Lin Y, Ortiz-Vega S, Yonezawa K, Avruch J. 2005. Rheb binds and regulates the mTOR kinase. Curr Biol 15: 702-713.

Ma XM, Blenis J. 2009. Molecular mechanisms of mTOR-mediated translational control. Nat Rev Mol Cell Biol 10: 307-318.

Magnuson B, Ekim B, Fingar DC. 2012. Regulation and function of ribosomal protein $\mathrm{S} 6$ kinase $(\mathrm{S} 6 \mathrm{~K})$ within $\mathrm{mTOR}$ signalling networks. Biochem J 441: 1-21.

Martin DP, Schmidt RE, DiStefano PS, Lowry OH, Carter JG, Johnson EM Jr. 1988. Inhibitors of protein synthesis and RNA synthesis prevent neuronal death caused by nerve growth factor deprivation. J Cell Biol 106: $829-844$.

Martin DP, Ito A, Horigome K, Lampe PA, Johnson EM Jr. 1992. Biochemical characterization of programmed cell death in NGFdeprived sympathetic neurons. J Neurobiol 23: 1205-1220.

Richter JD, Klann E. 2009. Making synaptic plasticity and memory last: Mechanisms of translational regulation. Genes Dev 23: 1-11.

Roizman B, Knipe DM, Whitley R. 2007. Herpes simplex viruses. In Fields' virology, 5th ed. (ed. DM Knipe, PM Howley), pp. 2501-2602. Lippincott, Williams \& Wilkins, Philadelphia.

Sengupta S, Peterson TR, Sabatini DM. 2010. Regulation of the mTOR complex 1 pathway by nutrients, growth factors, and stress. Mol Cell 40: $310-322$.

Taylor AM, Blurton-Jones M, Rhee SW, Cribbs DH, Cotman CW, Jeon NL. 2005. A microfluidic culture platform for CNS axonal injury, regeneration and transport. Nat Methods 2: 599-605.

Thompson RL, Preston CM, Sawtell NM. 2009. De novo synthesis of VP16 coordinates the exit from HSV latency in vivo. PLoS Pathog 5: e1000352. doi: 10.1371/journal.ppat.1000352.

Thoreen CC, Kang SA, Chang JW, Liu Q, Zhang J, Gao Y, Reichling LJ, Sim T, Sabatini DM, Gray NS. 2009. An ATP-competitive mammalian target of rapamycin inhibitor reveals rapamycin-resistant functions of mTORC1. J Biol Chem 284: 8023-8032.

Umbach JL, Kramer MF, Jurak I, Karnowski HW, Coen DM, Cullen BR. 2008. MicroRNAs expressed by herpes simplex virus 1 during latent infection regulate viral mRNAs. Nature 454: 780-783.

Wagner EK, Bloom DC. 1997. Experimental investigation of herpes simplex virus latency. Clin Microbiol Rev 10: 419-443.

Walsh D, Mohr I. 2011. Viral subversion of the host protein synthesis machinery. Nat Rev Microbiol 9: 860-875.

Wilcox CL, Johnson EM Jr. 1987. Nerve growth factor deprivation results in the reactivation of latent herpes simplex virus in vitro. J Virol 61: 2311-2315.

Wilcox CL, Smith RL, Freed CR, Johnson EM Jr. 1990. Nerve growth factor-dependence of herpes simplex virus latency in peripheral sympathetic and sensory neurons in vitro. J Neurosci 10: 1268-1275.

Willis DE, Twiss JL. 2006. The evolving roles of axonally synthesized proteins in regeneration. Curr Opin Neurobiol 16: 111-118.

Zheng JQ, Kelly TK, Chang B, Ryazantsev S, Rajasekaran AK, Martin KC, Twill JL. 2001. A functional role for intra-axonal protein synthesis during axonal regeneration from adult sensory neurons. J Neurosci 21: 9291-9303. 


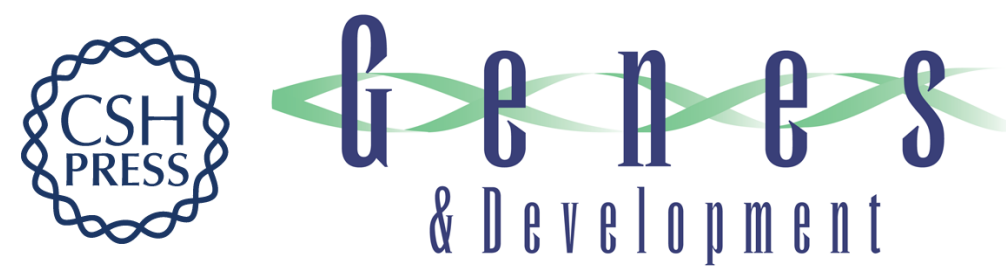

\section{Control of viral latency in neurons by axonal mTOR signaling and the 4E-BP translation repressor}

Mariko Kobayashi, Angus C. Wilson, Moses V. Chao, et al.

Genes Dev. 2012, 26:

Access the most recent version at doi:10.1101/gad.190157.112

\section{Supplemental http://genesdev.cshlp.org/content/suppl/2012/07/13/26.14.1527.DC1 Material}

Related Content

References

\section{License}

Email Alerting

Service
This article cites 40 articles, 16 of which can be accessed free at:

http://genesdev.cshlp.org/content/26/14/1527.full.html\#ref-list-1

Articles cited in:

http://genesdev.cshlp.org/content/26/14/1527.full.html\#related-urls

Connecting Stress to Viral Reactivation

Annalisa M. VanHook

Sci. Signal. July, 2012 5: ec194

Receive free email alerts when new articles cite this article - sign up in the box at the top right corner of the article or click here.

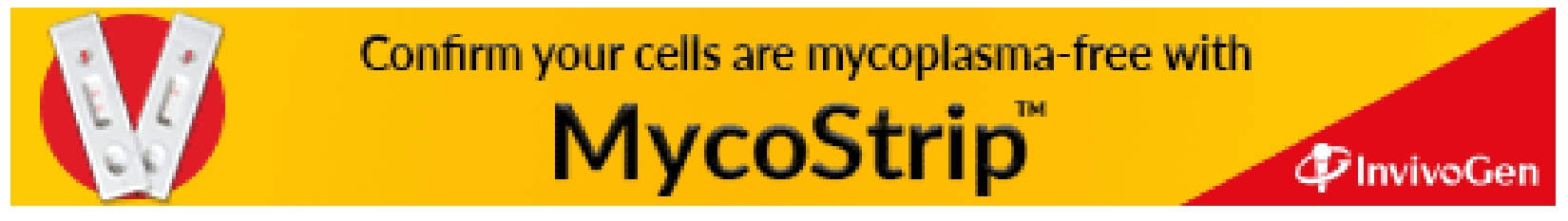

